\title{
The relationship of platelet-to-lymphocyte ratio with cognitive decline in T2DM
}

\author{
Licheng Du ${ }^{1 \dagger}$, Xueting $\mathrm{Hu}^{1,2 \dagger}$, Beibei Zhang ${ }^{3 \dagger}$, Xiaqi Miao ${ }^{1,2}$, Jianing Wang ${ }^{1,2}$, Jiamin Shen ${ }^{1,2}$, Keke Ding ${ }^{1,2}$, \\ Tian Zeng ${ }^{1,2}$, Fangyue Sun ${ }^{1,2}$, Hong Yang ${ }^{1 *}$ and Hai Lin ${ }^{1 *}$ (D)
}

\begin{abstract}
Purpose: We aimed to investigate the role of platelet-to-lymphocyte ratio (PLR) in cognitive decline in patients with type 2 diabetes mellitus (T2DM).

Methods: A total number of 261 T2DM patients were enrolled in this study. The T2DM patients were divided into two groups according to the median of PLR (PLR $<96.5, n=130 ; \mathrm{PLR} \geq 96.5, n=131)$. Cognitive impairment was defined as Mini-mental State Examination score $\leq 26$. Student's $t$ test and Chi-square test were used to test the difference between the groups, and logistics regression analysis were performed to verify whether high PLR was an independent factor for cognitive impairment.

Results: T2DM patients with cognitive impairment had significantly higher PLR level when compared with the simple diabetes group $(p=0.003)$. Incidence of cognitive impairment was higher in the high PLR group, compared to low PLR group $(p=0.040)$. Multivariate logistic regression analysis suggested that PLR was a risk biomarker of cognitive decline in T2DM patients (odds ratio $[\mathrm{OR}]=1.010,95 \% \mathrm{Cl}: 1.001-1.018, p=0.013$ ).

Conclusions: We demonstrated that a higher PLR was associated with cognitive decline in T2DM patients. The PLR may help to identify high-risk patients in time and provide clues for further prevention of cognitive dysfunction in T2DM patients.
\end{abstract}

\section{Introduction}

With the accelerated pace of global population aging, type 2 diabetes mellitus (T2DM), one of the most common diseases among the elderly, has attracted more and more attention. Many studies have demonstrated that the negative effects of T2DM on cognitive function have significant clinical significance $[1,2]$. In the course of diabetes, patients are more likely to progress to cognitive impairment and dementia, both can seriously affect the

\footnotetext{
*Correspondence: yanghong7115@yeah.net; linhairy@126.com

${ }^{\dagger}$ Co-first author: Licheng Du, Xueting Hu and Beibei Zhang

1 Department of Endocrinology, The Third Affiliated Hospital of Wenzhou

Medical University, NO.108 Wansong Road, Wenzhou 325000, Zhejiang, China

Full list of author information is available at the end of the article

Co-first author: Licheng Du, Xueting Hu and Beibei Zhang
}

patients' self-management, further exacerbating the disease and leading to more complications $[1,3]$. Therefore, studies on risk factors for cognitive decline in T2DM patients have been carried out extensively. In the same way, we are eager to find a biomarker, which are accurate, reliable and easily accessible.

Clinical studies have shown that patients with T2DM are often accompanied by increased concentrations of various inflammatory factors and inflammatory markers that can predict the occurrence of T2DM, as well as induce or worsen diabetes. Inflammation has been reported to play a role in pathogenesis as one of the causes of cognitive dysfunction or dementia. The ratio of platelet-to-lymphocyte ratio (PLR) is a wellknown marker of systemic inflammation [4, 5]. It has been studied as a biomarker of inflammation, showing great prognostic value as well as traditional markers of 
inflammation [6]. Therefore, in this study, we aimed to assess the relationship between PLR, which can be easily obtained from blood cell counts, and cognitive decline in patients with T2DM.

\section{Materials and methods Study population}

From September to December 2018, we conducted a cross-sectional study of 300 patients with T2DM in the Third Affiliated Hospital of Wenzhou Medical University. The exclusion criteria were as follows: (1) hypoglycemic coma, diabetic ketoacidosis and other acute complications of diabetes $(n=2)$; (2) severe hepatic and renal insufficiency, severe systemic disease (Malignant tumor, thyroid disease, severe infection, severe anemia, etc.) (n =11); (3) acute cardiovascular events, Parkinson's disease, epilepsy, moderate depression or other mental disorders $(n=21)$; (4) severe loss of sight or hearing $(n=2)$; (5) missing data $(n=3)$. Finally, there were 261 patients left after excluding 39 patients who met the criteria. The research was approved by the Ethics Committee of the Third Affiliated Hospital of Wenzhou Medical University and obtained informed consent from all patients.

\section{Data collection}

We collected demographic information of the patients through face-to-face questioning to gather information such as age, sex, years of education, smoking history, alcohol consumption history, diabetes course, etc. We performed a blood routine examination of the patients and obtained indicators like glycosylated hemoglobin (HbA1c), white blood cell (WBC), red blood cell (RBC), hemoglobin $(\mathrm{Hb})$, triglyceride (TG), total cholesterol (TC), high density lipoprotein (HDL), low density lipoprotein (LDL), platelet (PLT), lymphocytes. WBC, RBC, $\mathrm{Hb}, \mathrm{HbA1c}$, PLT, lymphocytes were counted using XT1800i (Sysmex, Kobe, Japan). TG, TC, HDL, LDL were counted using ARCHITECT c16000 (Abbott Laboratories, Illinois, USA). Platelet-to-lymphocyte ratio (PLR) was calculated as platelet/lymphocyte. What's more, we assessed the cognitive status of all patients through Minimental State Examination (MMSE), which is one of the most widely used scales to assess whether people have cognitive impairment or not. In this study, diabetics were divided into two groups according to the MMSE score: the cognitive-unimpaired group (MMSE $>26, n=190$ ) and the cognitive-impaired group (MMSE $\leq 26, n=71$ ) $[7,8]$.

\section{Statistical analysis}

All statistical analyses were performed in IBM SPSS Statistics 25.0 and all the figures were drawn by Graphpad Prism 7.0. The continuous variables of normal distribution were described by mean \pm standard deviation and using Student's $t$ test to analyze the differences between groups. Continuous variables with non-normal distribution were represented by median and interquartile range of continuous variables, and the difference between groups was analyzed by Mann-Whitney $U$ test. The categorical variables were described by counts or percentage, and Chi-square test was used for the comparison between groups. Univariate Logistics regression analysis was used to study the variables related to cognitive status of patients with T2DM. Multivariate logistic regression analysis was used to investigate the effect of PLR on the cognitive status of diabetic patients after controlling for confounding factors. $p<0.05$ in all analyses was considered statistically significant.

\section{Result}

\section{Baseline characteristics of the study subjects}

Among the 261 diabetic patients, 190 were cognitiveunimpaired while 71 were cognitive-impaired. The baseline characteristics between groups with and without cognitive impairment were presented in Table 1 . There were significant differences between the two groups in age $(p<0.001)$, sex $(p<0.001)$, education $(p<0.001)$, hypertension $(p=0.016)$, smoking $(p<0.001)$, drinking $(p=0.025), \mathrm{RBC}(p=0.001), \mathrm{Hb}(p<0.001), \mathrm{TC}(p=$ $0.025)$ and PLR $(p=0.002)$. The cognitive impairment group was older, having higher proportion of women, prevalence of hypertension and higher level of PLR, but lower in the other respects than the non-cognitive impairment group. We can intuitively see that PLR in the cognitive impairment group was significantly higher than that in the non-cognitive impairment group (Fig. 1).

\section{The association of PLR with cognitive function}

Then all the diabetic patients were divided into G1 (PLR $<96.5, n=130$ ) and G2 (PLR $\geq 96.5, n=131$ ) groups according to the median of PLR. As shown in Table 2, diabetic duration $(p=0.002)$ and age $(p=0.034)$ in G2 group was significantly higher than that in G1 group. Apart from this, the figures of G2 group were lower than the G1 group in the other statistically significant indicators like sex $(p<0.001)$, education $(p=0.015)$, smoking $(p<0.001)$, drinking $(p<0.001), \mathrm{HbA1c}(p=0.036), \mathrm{RBC}$ $(p<0.001), \mathrm{Hb}(p<0.001), \mathrm{TG}(p=0.001)$ and TC $(p=$ $0.020)$.

\section{Increased PLR level is related to cognitive decline}

In order to explore the distribution of patients with cognitive impairment in different concentration of PLR, we divided the patients into two groups through the median of PLR. 32.8\% patients suffered from cognitive impairment in the group with a high PLR level (PLR $\geq$ 
Table 1 Demographic and laboratory characteristics of simple diabetic patients and diabetic patients with cognitive impairment

\begin{tabular}{|c|c|c|c|}
\hline Characteristics & Without $\mathrm{Cl}(n=190)$ & With Cl $(n=71)$ & $p$ \\
\hline Age, (years) & $55(48-61)$ & $60(56-67)$ & $<0.001$ \\
\hline Sex, (male, n\%) & $141(74.2)$ & $31(43.6)$ & $<0.001$ \\
\hline BMl & $24.0(22.0-26.6)$ & $24.0(22.5-26.7)$ & 0.844 \\
\hline Education, (years) & $7(4-9)$ & $0(0-2)$ & $<0.001$ \\
\hline Diabetes duration, (years) & $8(3-11)$ & $9(5-16)$ & 0.127 \\
\hline Hypertension, n (\%) & $73(38.4)$ & $39(54.9)$ & 0.016 \\
\hline Hyperlipidemia, n (\%) & $53(27.8)$ & $20(28.1)$ & 0.914 \\
\hline Smoking, n (\%) & $111(58.4)$ & $21(29.5)$ & $<0.001$ \\
\hline Drinking, n (\%) & $96(50.5)$ & $25(35.2)$ & 0.025 \\
\hline $\mathrm{HbA1c}$ & $9.38(7.96-11.28)$ & $9.45(7.83-11.33)$ & 0.773 \\
\hline $\mathrm{WBC},\left(\times 10^{9} / \mathrm{L}\right)$ & $6.10(5.10-7.20)$ & $6.10(5.30-7.40)$ & 0.614 \\
\hline $\mathrm{RBC},\left(\times 10^{12} / \mathrm{L}\right)$ & $4.60(4.32-4.90)$ & $4.47(4.15-4.71)$ & 0.001 \\
\hline $\mathrm{Hb},(\mathrm{g} / \mathrm{L})$ & $140.9 \pm 14.73$ & $55.02(47.96-61.26)$ & $<0.001$ \\
\hline $\mathrm{TG},(\mathrm{mmol} / \mathrm{L})$ & $1.60(1.06-2.35)$ & $1.62(1.14-2.26)$ & 0.675 \\
\hline $\mathrm{TC},(\mathrm{mmol} / \mathrm{L})$ & $4.81(3.86-5.52)$ & $4.38(3.69-5.01)$ & 0.025 \\
\hline $\mathrm{HDL},(\mathrm{mmol} / \mathrm{L})$ & $0.97(0.84-1.18)$ & $1.02(0.85-1.16)$ & 0.953 \\
\hline $\mathrm{LDL},(\mathrm{mmol} / \mathrm{L})$ & $2.97(2.22-3.58)$ & $2.53(2.11-3.30)$ & 0.073 \\
\hline PLR & $92.94(76.00-118.82)$ & 111.82(84.62-146.11) & 0.002 \\
\hline
\end{tabular}

$C l$ cognitive impairment, $B M I$ body mass index, HbAlc glycosylated hemoglobin, WBC white blood cells, $R B C$ red blood cells, $H b$ hemoglobin, $T G$ triglyceride, $T C$ total cholesterol, $H D L$ high density lipoprotein, $L D L$ low density lipoprotein, $P L R$ platelet-to-lymphocyte ratio

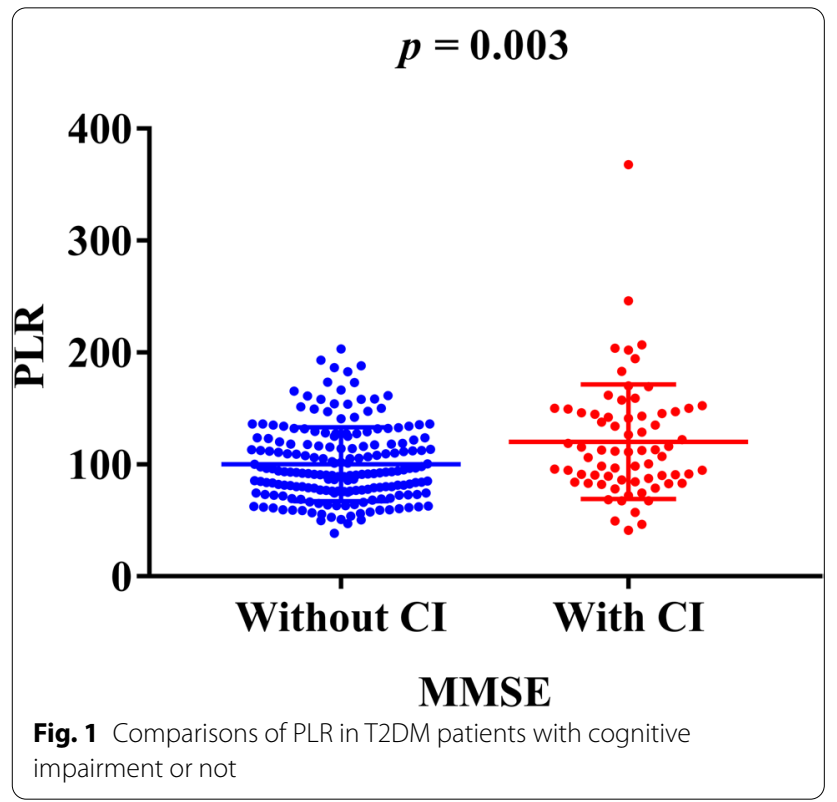

96.5) while only $21.5 \%$ patients suffered from cognitive impairment in the group with a low PLR level $(p=0.040)$ (Fig. 2). In addition, PLR and MMSE scores were found to be correlated through linear regression analysis $(r=$ $-0.250, p<0.001$ ) (Fig. 3).

To further explore the relationship between PLR and cognitive impairment in diabetic patients, we
Table 2 Characteristics of patients with diabetes mellitus with cognitive impairment according to PLR median

\begin{tabular}{|c|c|c|c|}
\hline Characteristics & $\begin{array}{l}\text { PLR }<96.5 \\
(n=130)\end{array}$ & $\begin{array}{l}\text { PLR } \geq 96.5 \\
(n=131)\end{array}$ & $p$ \\
\hline With Cl, n (\%) & $28(21.5)$ & $43(32.8)$ & 0.040 \\
\hline Age (years) & $55(49-61)$ & $58(52-64)$ & 0.034 \\
\hline Sex (male, n\%) & $99(76.1)$ & $73(55.7)$ & $<0.001$ \\
\hline $\mathrm{BMI}$ & $24.2(22.3-26.8)$ & $23.6(21.9-26.0)$ & 0.060 \\
\hline Education (years) & $6(2-9)$ & $5(0-8)$ & 0.015 \\
\hline Diabetes duration (years) & $6(2-11)$ & $9(5-16)$ & 0.002 \\
\hline Hypertension, n (\%) & $56(43.0)$ & $56(42.7)$ & 0.957 \\
\hline Hyperlipidemia, n (\%) & 35 (26.9) & $38(29.0)$ & 0.736 \\
\hline Smoking, n (\%) & $83(63.8)$ & $49(37.4)$ & $<0.001$ \\
\hline Drinking, n (\%) & $75(57.6)$ & $46(35.1)$ & $<0.001$ \\
\hline $\mathrm{HbA1c}$ & $9.71(8.41-11.45)$ & $9.27(7.46-10.90)$ & 0.036 \\
\hline WBC $\left(\times 10^{9} / \mathrm{L}\right)$ & $6.30(5.50-7.30)$ & $5.85(5.00-7.20)$ & 0.052 \\
\hline $\mathrm{RBC}\left(\times 10^{12} / \mathrm{L}\right)$ & $4.71(4.46-4.96)$ & $4.41(4.14-4.64)$ & $<0.001$ \\
\hline $\mathrm{Hb}(\mathrm{g} / \mathrm{L})$ & $143.59 \pm 14.58$ & $133.07 \pm 14.30$ & $<0.001$ \\
\hline $\mathrm{TG}(\mathrm{mmol} / \mathrm{L})$ & $1.73(1.25-2.76)$ & $1.45(0.96-2.14)$ & 0.001 \\
\hline $\mathrm{TC}(\mathrm{mmol} / \mathrm{L})$ & $4.83(4.05-5.56)$ & $4.60(3.72-5.31)$ & 0.020 \\
\hline $\mathrm{HDL}(\mathrm{mmol} / \mathrm{L})$ & $0.96(0.83-1.15)$ & $1.02(0.88-1.21)$ & 0.167 \\
\hline LDL (mmol/L) & $2.96(2.24-3.61)$ & $2.74(2.08-3.50)$ & 0.279 \\
\hline
\end{tabular}

Cl cognition impairment, BMI body mass index, HbAlc glycosylated hemoglobin, WBC white blood cells, $R B C$ red blood cells, $H b$ hemoglobin, $T G$ triglyceride, $T C$ total cholesterol, $H D L$ high density lipoprotein, $L D L$ low density lipoprotein 

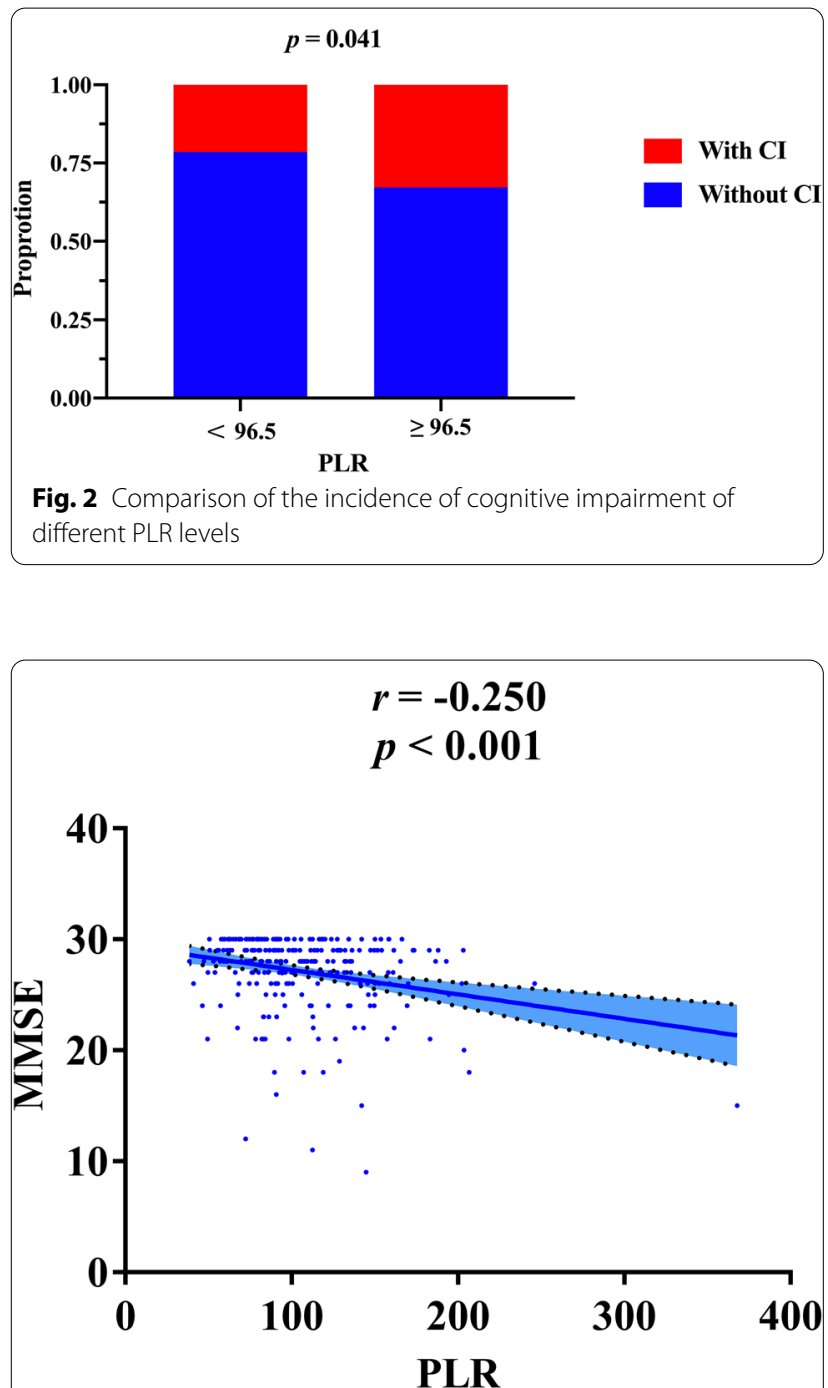

Fig. 3 Correlation between PLR and MMSE. $r=-0.250, p<0.001$

performed a single-factor regression analysis. As shown in Table 3, age $(p<0.001)$, sex $(p<0.001)$, education $(p$ $<0.001)$, hypertension $(p=0.017)$, smoking $(p<0.001)$, drinking $(p=0.026), \operatorname{RBC}(p=0.004), \mathrm{Hb}(p<0.001)$, PLR $(p=0.001)$ were significantly correlated with cognitive impairment in diabetic patients.

Multivariate logistic regression analyses were performed to control other potential confounding variables (Table 4). In Model 1, nothing was adjusted (odds ratio $[\mathrm{OR}]=1.013,95 \% \mathrm{CI}: 1.005-1.020, p=0.001$ ). After adjusting for age, sex in Model 2, the linkage between PLR and cognitive impairment remained significant (odds ratio $[\mathrm{OR}]=1.010,95 \%$ CI: $1.002-1.018$, $p=0.014$ ). On the basis of Model 2, we additionally made adjustments for smoking and drinking in Model 3, the linkage between PLR and cognitive impairment
Table 3 Univariate logistic regression analyses for diabetes mellitus with cognitive impairment

\begin{tabular}{|c|c|c|c|}
\hline \multirow[t]{2}{*}{ Variables } & \multicolumn{3}{|c|}{ Univariate logistic regression } \\
\hline & OR & $95 \% \mathrm{Cl}$ & $p$ \\
\hline Age, (years) & 1.083 & $1.047-1.119$ & $<0.001$ \\
\hline Sex, (male, n\%) & 3.713 & $2.098-6.570$ & $<0.001$ \\
\hline BMI & 0.976 & $0.894-1.065$ & 0.582 \\
\hline Education, (years) & 0.635 & $0.562-0.717$ & $<0.001$ \\
\hline Diabetes duration, (years) & 1.031 & $0.993-1.072$ & 0.115 \\
\hline Hypertension, n (\%) & 0.512 & $0.295-0.889$ & 0.017 \\
\hline Hyperlipidemia, n (\%) & 0.967 & $0.527-1.776$ & 0.914 \\
\hline Smoking, n (\%) & 3.388 & $1.885-6.089$ & $<0.001$ \\
\hline Drinking, n (\%) & 1.899 & $1.080-3.340$ & 0.026 \\
\hline $\mathrm{HbA} 1 \mathrm{c}$ & 0.992 & $0.880-1.118$ & 0.893 \\
\hline WBC $\left(\times 10^{9} / L\right)$ & 1.040 & $0.896-1.207$ & 0.607 \\
\hline $\mathrm{RBC},\left(\times 10^{12} / \mathrm{L}\right)$ & 0.428 & $0.241-0.760$ & 0.004 \\
\hline $\mathrm{Hb},(\mathrm{g} / \mathrm{L})$ & 0.958 & $0.939-0.977$ & $<0.001$ \\
\hline $\mathrm{TG},(\mathrm{mmol} / \mathrm{L})$ & 1.024 & $0.977-1.073$ & 0.325 \\
\hline $\mathrm{TC},(\mathrm{mmol} / \mathrm{L})$ & 1.003 & $0.882-1.139$ & 0.969 \\
\hline $\mathrm{HDL},(\mathrm{mmol} / \mathrm{L})$ & 0.944 & $0.382-2.330$ & 0.900 \\
\hline LDL, (mmol/L) & 0.768 & $0.565-1.044$ & 0.092 \\
\hline PLR & 1.013 & $1.005-1.020$ & 0.001 \\
\hline
\end{tabular}

$B M I$ body mass index, $H b A 1 C$ glycosylated hemoglobin, WBC white blood cells, $R B C$ red blood cells, $H b$ hemoglobin, $T G$ triglyceride, $T C$ total cholesterol, $H D L$ high density lipoprotein, $L D L$ low density lipoprotein, $P L R$ platelet-tolymphocyte ratio

Table 4 Adjusted Odds Ratio (95\% Confidence Interval) for T2DM with cognitive impairment

\begin{tabular}{llll}
\hline Variables & OR & $\mathbf{9 5 \%} \mathrm{Cl}$ & $\boldsymbol{p}$ \\
\hline Model 1 & 1.013 & $1.005-1.020$ & 0.001 \\
Model 2 & 1.010 & $1.002-1.018$ & 0.014 \\
Model 3 & 1.010 & $1.002-1.018$ & 0.013 \\
\hline
\end{tabular}

Model 1 is univariate analysis

Model 2 is adjusted by age and sex

Model 3 is adjusted by age, sex, smoking and drinking

still remained significant (odds ratio $[\mathrm{OR}]=1.010,95 \%$ CI: $1.001-1.018, p=0.013)$. After two adjustments, there was still a correlation between PLR and cognitive impairment, which showed that PLR was probably an independent impact factor of T2DM patients with cognitive impairment.

\section{Discussion}

In this cross-sectional study, we investigated the relationship between PLR and cognitive decline in T2DM in 261 patients from the Third Affiliated Hospital of Wenzhou Medical University. The main findings of this study were as follows: (1) PLR was significantly correlated with 
cognitive function in patients with type 2 diabetes; (2) After adjusting for age, sex, smoking and alcohol consumption, the $p$ value of PLR was still significant. Our study found that PLR levels in T2DM patients combined with cognitive impairment were higher than those in simple T2DM patients.

As far as we know, T2DM is a chronic metabolic disease caused by insulin resistance and insufficient insulin secretion compensation response. Inflammatory pathways are considered as potential agents of diabetes [9]. The mechanisms that trigger inflammation in T2DM remain unclearly. Inflammation may promote the development of T2DM by causing insulin resistance, while hyperglycemia may exacerbate the inflammatory response, thus promoting the long-term complications of diabetes [10]. In the course of inflammatory response, the continuous production of pro-inflammatory factors such

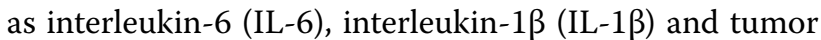
necrosis factor- $\alpha$ (TNF- $\alpha)[11,12]$ can cause various nervous system lesions, including amyloidosis, neuronal death $[13,14]$, cortical thinning $[15,16]$, reduced brain volume [15], cerebral vascular disease related events such as micro hemorrhage, infarcts $[17,18]$ and neurodegeneration [2]. These lesions can lead to cognitive decline.

PLR, which represents the balance between platelet and lymphocyte levels, having been recognized as an indicator of inflammatory status in patients with a variety of chronic inflammatory diseases [19]. Baodong Qin et al. [20] showed that PLR level was significantly increased in patients with rheumatic diseases compared with normal subjects. Meanwhile, Guang Shi et al. [21] reported that PLR of asthmatic critically ill patients was significantly higher than that of non-critically ill patients and control group. Apart from this, van der Willik et al. [22] found that PLR levels were associated with lower cognitive performance in the study of breast cancer survivors. In our study, PLR of patients with cognitive impairment was significantly higher than that of patients with type 2 diabetes alone. Studies have found that high levels of PLR are associated with activity and poor prognosis in a variety of diseases, including chronic atrophic gastritis [23], colorectal cancer [24] and osteosarcoma [25]. PLR may be more reliable as a combination of these two markers due to the complex interaction between platelets and lymphocytes. And it is a very easy indicator to be calculated.

Our study has some certain limitations. Firstly, this is a single-center cross-sectional study, with the possibility of selection bias, and the generalization of the findings to clinical fields should be cautious. Secondly, the sample size is relatively small. In addition, the MMSE score alone may not be comprehensive enough to assess patients' cognitive status. Thirdly, certain drugs have effects on cognitive impairment, but this was not included in our data analysis. Finally, we only discussed the clinical significance of PLR and cognitive decline in T2DM patients, lacking the research on the specific mechanism. Therefore, in future studies, prospective cohort studies should be designed to expand the scope and number of sample collection, combined with more indicators to evaluate cognitive function, and further study the mechanism of PLR in the process of cognitive decline in T2DM patients, so as to provide greater referential significance for clinical work.

\section{Conclusions}

We demonstrated that a higher PLR level was associated with cognitive decline in T2DM patients. The PLR may help to identify high-risk patients in time and provide clues for further prevention of cognitive dysfunction in T2DM patients.

\section{Acknowledgements}

We sincerely thank the participating hospitals, diabetic patients, their families and colleagues who have supported this study.

\section{Authors' contributions}

$\mathrm{HL}, \mathrm{HY}$ participated in the conception and design; $\mathrm{LD}, \mathrm{XH}, \mathrm{BZ}, \mathrm{XM}, J \mathrm{~W}$, JS, $K D, T Z, F S$ are responsible for data acquisition; $L D, X H, B Z, X M$ participated in data analysis and interpretation; $L D, X H, B Z$ are responsible for drafting the article; HL, HY, LD, XH, BZ, XM, JW, JS, KD, TZ, FS are responsible for critically revising the article for important intellectual content. All the authors read and approved the final manuscript.

\section{Funding}

Not applicable.

\section{Data Availability}

Data included in the current study are not publicly available to ensure confidentiality of the patients but are available from the corresponding author on reasonable request.

\section{Code Availability \\ Not applicable.}

\section{Declarations}

Ethics approval and consent to participate

This study was approved by the Ethics Committee of the Third Affiliated Hospital of Wenzhou Medical University and was carried out in accordance with the Declaration of Helsinki. Because this study was retrospectively designed, the ethics committee granted a waiver of the requirement for informed consent for analyzing existing data in accordance with the national legislation and the institutional requirements. Personal identification information was anonymized and replaced with a coding system in this study.

\section{Consent for publication}

Not applicable.

\section{Competing interests}

The authors report no declarations of interest.

\section{Author details}

${ }^{1}$ Department of Endocrinology, The Third Affiliated Hospital of Wenzhou Medical University, NO.108 Wansong Road, Wenzhou 325000, Zhejiang, China. ${ }^{2}$ School of the First Clinical Medical Sciences, Wenzhou Medical University, Wenzhou, China. ${ }^{3}$ Department of Gastroenterology, The Third Affiliated Hospital of Wenzhou Medical University, Wenzhou, China. 
Received: 2 October 2021 Accepted: 15 December 2021

Published online: 24 December 2021

\section{References}

1. Salama I, et al. Plasma microRNAs biomarkers in mild cognitive impairment among patients with type 2 diabetes mellitus. PloS one. 2020;15(7):e0236453.

2. Xia S, et al. The factors contributing to cognitive dysfunction in type 2 diabetic patients. Annals of translational medicine. 2020;8(4):104.

3. Munshi M. Cognitive dysfunction in older adults with diabetes: what a clinician needs to know. Diabetes Care. 2017:40(4):461-7.

4. He Q, et al. The relationship between inflammatory marker levels and hepatitis C virus severity. Gastroenterol Res Pract. 2016;2016:2978479.

5. Kumarasamy C, et al. Prognostic significance of blood inflammatory biomarkers NLR, PLR, and LMR in cancer-a protocol for systematic review and meta-analysis. Medicine. 2019;98(24):e14834

6. Balta S, Ozturk C. The platelet-lymphocyte ratio: A simple, inexpensive and rapid prognostic marker for cardiovascular events. Platelets. 2015;26(7):680-1.

7. Ueda M, et al. Association of orthostatic blood pressure with the symptoms of orthostatic hypotension and cognitive impairment in patients with multiple system atrophy. J Clin Neurosci. 2020;75:40-4.

8. Ambrosini $\mathrm{E}$, et al. Automatic speech analysis to early detect functional cognitive decline in elderly population. In: Annual international conference of the IEEE engineering in medicine and biology Society. IEEE Engineering in Medicine and Biology Society. Annual International Conference; 2019. 2019: p. 212-216.

9. Shoelson S, Lee J, Goldfine A. Inflammation and insulin resistance. J Clin Investig. 2006;116(7):1793-801.

10. Lontchi-Yimagou E, et al. Diabetes mellitus and inflammation. Curr Diabetes Rep. 2013;13(3):435-44.

11. Heppner F, Ransohoff R, Becher B. Immune attack: the role of inflammation in Alzheimer disease. Nat Rev Neurosci. 2015;16(6):358-72.

12. Sastre M, Klockgether T, Heneka M. Contribution of inflammatory processes to Alzheimer's disease: molecular mechanisms. Int J Dev Neurosci. 2006;24:167-76

13. Akiyama $\mathrm{H}$, et al. Inflammation and Alzheimer's disease. Neurobiol Aging. 2000;21(3):383-421.

14. Block M, Zecca L, Hong J. Microglia-mediated neurotoxicity: uncovering the molecular mechanisms. Nat Rev Neurosci. 2007;8(1):57-69.

15. GuY, et al. Circulating inflammatory biomarkers in relation to brain structural measurements in a non-demented elderly population. Brain Behav Immun. 2017;65:150-60.

16. McCarrey A, et al. Interleukin-6 is linked to longitudinal rates of cortical thinning in aging. Transl Neurosci. 2014;5(1):1-7.

17. GuY, et al. Circulating inflammatory biomarkers are related to cerebrovascular disease in older adults. Neurol(R) Neuroimmunol Neuroinflamm. 2019;6(1):e521.

18. Swardfager W, et al. Peripheral inflammatory markers indicate microstructural damage within periventricular white matter hyperintensities in Alzheimer's disease: a preliminary report, vol. 7. Amsterdam: Alzheimer's \& dementia; 2017. p. 56-60.

19. Sung $P$, et al. The correlation between severity of neurological impairment and left ventricular function in patients after acute ischemic stroke. J Clin Med. 2019;8(2):190.

20. Qin B, et al. Neutrophil to lymphocyte ratio (NLR) and platelet to lymphocyte ratio (PLR) were useful markers in assessment of inflammatory response and disease activity in SLE patients. Mod Rheumatol. 2016:26(3):372-6.

21. Shi G, Zhao J, Ming L. Clinical significance of peripheral blood neutrophillymphocyte ratio and platelet- lymphocyte ratio in patients with asthma Nan fang yi ke da xue xue bao J South Med Univ. 2017:37(1):84-8.

22. van der Willik K, et al. Inflammation markers and cognitive performance in breast cancer survivors 20 years after completion of chemotherapy: a cohort study. Breast Cancer Res BCR. 2018;20(1):135.

23. Du Y, et al. Chronic gastritis in China: a national multi-center survey. BMC Gastroenterol. 2014;14:21.
24. Emir S, et al. Comparison of colorectal neoplastic polyps and adenocarcinoma with regard to NLR and PLR. Eur Rev Med Pharmacol Sci. 2015:19(19):3613-8.

25. Xia W, et al. Prognostic performance of pre-treatment NLR and PLR in patients suffering from osteosarcoma. World J Surg Oncol. 2016;14:127.

\section{Publisher's Note}

Springer Nature remains neutral with regard to jurisdictional claims in published maps and institutional affiliations.
Ready to submit your research? Choose BMC and benefit from:

- fast, convenient online submission

- thorough peer review by experienced researchers in your field

- rapid publication on acceptance

- support for research data, including large and complex data types

- gold Open Access which fosters wider collaboration and increased citations

- maximum visibility for your research: over $100 \mathrm{M}$ website views per year

At BMC, research is always in progress.

Learn more biomedcentral.com/submissions 